\title{
Wind and boundary layers in Rayleigh-Bénard convection. II. Boundary layer character and scaling
}

\author{
Maarten van Reeuwijk, ${ }^{1, *}$ Harm J. J. Jonker, ${ }^{2}$ and Kemo Hanjalic ${ }^{2,3}$ \\ ${ }^{1}$ Department of Civil and Environmental Engineering, Imperial College London, \\ Imperial College Road, London, SW7 2AZ, United Kingdom \\ ${ }^{2}$ Department of Multiscale Physics and J. M. Burgers Center for Fluid Dynamics, Delft University of Technology, \\ Lorentzweg 1, 2628 CJ Delft, The Netherlands \\ ${ }^{3}$ Department of Mechanics and Aeronautics, University of Rome, "La Sapienza", Rome, Italy
}

(Received 3 September 2007; published 20 March 2008)

\begin{abstract}
The scaling of the kinematic boundary layer thickness $\lambda_{u}$ and the friction factor $C_{f}$ at the top and bottom walls of Rayleigh-Bénard convection is studied by direct numerical simulation (DNS). By a detailed analysis of the friction factor, a new parameterisation for $C_{f}$ and $\lambda_{u}$ is proposed. The simulations were made of an $L / H=4$ aspect-ratio domain with periodic lateral boundary conditions at $\operatorname{Ra}=\left\{10^{5}, 10^{6}, 10^{7}, 10^{8}\right\}$ and $\operatorname{Pr}=1$. The continuous spectrum, as well as significant forcing due to Reynolds stresses, clearly indicates a turbulent character of the boundary layer, while viscous effects cannot be neglected, judging from the scaling of classical integral boundary layer parameters with Reynolds number. Using a conceptual wind model, we find that the friction factor $C_{f}$ should scale proportionally to the thermal boundary layer thickness as $C_{f} \propto \lambda_{\Theta} / H$, while the kinetic boundary layer thickness $\lambda_{u}$ scales inversely proportionally to the thermal boundary layer thickness and wind Reynolds number $\lambda_{u} / H \propto\left(\lambda_{\Theta} / H\right)^{-1} \mathrm{Re}^{-1}$. The predicted trends for $C_{f}$ and $\lambda_{u}$ are in agreement with DNS results.
\end{abstract}

DOI: 10.1103/PhysRevE.77.036312

PACS number(s): 47.20.Bp, 47.27.nb, 44.25.+f

\section{INTRODUCTION}

The structure of the boundary layer is of great importance for understanding the turbulent heat transfer characteristics of Rayleigh-Bénard convection. Inherently unstable due to buoyancy effects, the thermal boundary layer with thickness $\lambda_{\Theta}$ is in a dynamic equilibrium of heating (cooling) by thermal diffusion and the detrainment (entrainment) of heat due to impinging and ejecting thermals at the bottom (top) plate. This process creates large temperature gradients across the boundary layer, thereby enhancing the heat transfer through the wall and thus the Nusselt number Nu. Next to a thermal boundary layer, one can identify a kinematic boundary layer with thickness $\lambda_{u}$, associated with the velocity field. Depending on the Prandtl number $\operatorname{Pr}=\nu \kappa^{-1}$, which is the ratio between the kinematic viscosity $\nu$ and thermal diffusivity $\kappa$, the kinematic boundary layer can be nested inside the thermal boundary layer or vice versa, which influences the effectiveness of the heat transfer as a function of the Rayleigh number $\mathrm{Ra}$. The Rayleigh number $\mathrm{Ra}$ is defined as $\mathrm{Ra}$ $=\beta g \Delta \Theta H^{3}(\nu \kappa)^{-1}$, where $\beta$ is the thermal expansion coefficient, $g$ the gravitational constant, $\Delta \Theta$ the temperature difference between the top and bottom plates, and $H$ the domain height. The scalings of $\lambda_{\Theta}$ and $\lambda_{u}$ as a function of Ra and $\operatorname{Pr}$ are therefore of importance for proper prediction of the heat transfer.

In the theory of Grossmann and Lohse [1], the wind velocity $U$ and the boundary layer thicknesses $\lambda_{u}$ and $\lambda_{\Theta}$ are central parameters, which are used to estimate the dissipation rates of kinematic energy and temperature variance in the bulk and the boundary layers. In the theory, $\lambda_{\Theta}$ and $\lambda_{u}$ are defined as

\footnotetext{
*m.vanreeuwijk@imperial.ac.uk
}

$$
\begin{gathered}
\lambda_{\Theta} \propto H /(2 \mathrm{Nu}), \\
\lambda_{u} \propto H \mathrm{Re}^{-1 / 2} .
\end{gathered}
$$

While (1) holds excellently, the correspondence of (2) with experiments [2,3] and simulations [4,5] is less satisfactory. Relation (2) can be obtained by nondimensionalizing the steady laminar two-dimensional Prandtl boundary layer equations [6,7], from which (2) follows immediately. However, the measured Re dependence of $\lambda_{u}$ is much weaker than predicted by (2) (see also Fig. 1). It has been suggested that the difference is due to geometry effects [8] (plate-filling vs laterally restricted flow).

In this paper, we argue that the disparity between the expected and the observed scaling of $\lambda_{u}$ is because the top and bottom boundary layers are not laminar, i.e., forcing due to Reynolds stresses cannot be neglected in the kinematic

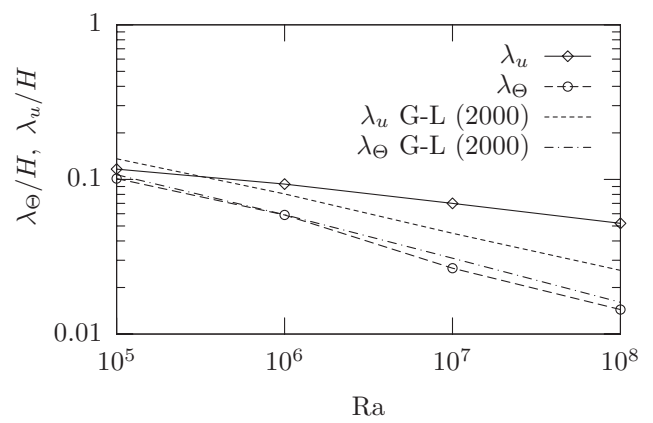

FIG. 1. Thermal and kinematic boundary layer thicknesses $\lambda_{\Theta}$ and $\lambda_{u}$ as a function of Ra. The dashed and dash-dotted lines in the graphs are predictions by the G-L theory for $\lambda_{u}$ and $\lambda_{\Theta}$, respectively. 
boundary layer. Consequently, the arguments leading to (2) do not hold. With a detailed direct numerical simulation (DNS) study of the momentum and heat budgets and the friction factor, and using the wind model of the accompanying paper [9], we derive new parametrizations for $\lambda_{u}$ and $C_{f}$.

A related question is whether or not the boundary layers can be regarded as turbulent. The Reynolds number Re is too low to sustain a "classical" turbulent boundary layer (Re $\approx 1500$ at $\mathrm{Ra}=10^{8}$ ), i.e., a boundary layer where the turbulence production due to shear is in local equilibrium with dissipation. Hence, the general view is that the boundary layers are laminar, but time dependent. Although time dependence due to plume impingement and detachment prevents laminarity in the strict sense, the assumption could be justified if the plumes are passive with respect to the scaling of integral boundary layer parameters such as the friction factor $C_{f}$ and the kinematic boundary layer thickness $\lambda_{u}$. Several other studies show that the friction factor scales similarly to a Blasius boundary layer [10-12]. However, the scaling of $\lambda_{u}$ does not comply with classical laminar scaling (2), as discussed before. Furthermore, a recent study of time spectra in the bottom kinematic boundary layer revealed that the spectra in the boundary layer and in the bulk were practically indistinguishable [13], a strong indication of turbulence. In order to understand this dual behavior, we study several turbulence indicators for the boundary layers, such as the spectra and the shape and friction factors.

The paper is outlined as follows. A brief summary of the code for direct simulation and symmetry-accounted ensemble averaging is given in Sec. II. The scaling of the boundary layer thickness, the velocity profile, the friction factor, and the shape factor are studied in Sec. III A, Sec. III B, and Sec. III C, respectively. Then, we study the space and time spectra (Sec. III D). In Sec. III E, the mean momentum and temperature budgets in the boundary layers are studied to clarify the importance of fluctuations in the boundary layers. Using the results from the momentum budgets, the friction factor $C_{f}$ is decomposed into a pressure and a momentum-flux contribution in Sec. III F. This leads to the insight that the main contribution is from the pressure gradient. Using the conceptual wind model derived in the accompanying paper [9], scaling laws for $C_{f}$ and $\lambda_{u}$ are derived in Sec. IV. As the results show that the flow has many typical features of turbulence but also of laminarity, the interpretation of the results is discussed in Sec. V. Conclusions are drawn in Sec. VI.

\section{SIMULATIONS}

Direct simulation of Rayleigh-Bénard convection has been performed at $\operatorname{Ra}=\left\{10^{5}, 10^{6}, 10^{7}, 10^{8}\right\}$ and $\operatorname{Pr}=1$ in a $\Gamma$ $=4$ aspect-ratio domain. The code is based on a second-order variance-preserving finite-difference discretization of the three-dimensional Navier-Stokes equations and is fully parallellized. For all simulations, a grid was used with sufficient resolution to resolve the smallest turbulent scales, i.e., the Kolmogorov scale $\eta_{K}=\left(\nu^{3} / \varepsilon\right)^{1 / 4}$ and the Corrsin scale $\eta_{C}$ $=\operatorname{Pr}^{-1 / 2} \eta_{K}$. The top and bottom walls are rigid (no slip) and of fixed temperature. At the side domain boundaries, periodic boundary conditions are applied. For each Ra except the highest, 400 independent realizations were obtained by performing ten independent simulations and sampling the velocity and temperature field roughly twice every convective turnover time. Because of the formidable computational requirements for $\mathrm{Ra}=10^{8}$, we use this simulation only for the results of Fig. 1 and confine the wind-decomposed analysis to the lower-Ra cases, though without loss of generality.

As in domains confined by sidewalls, a wind structure develops also in domains with lateral periodic boundary conditions. However, here the wind structure can be located anywhere in the domain since it is not kept in place by sidewalls. To extract the wind, symmetry-accounted ensemble averaging is used [14], which aligns the wind structure in different realizations before averaging. In this way a wind structure can be identified unambiguously for these domains, by which a decomposition into wind and fluctuations becomes possible. The resulting average velocity and temperature (threedimensional fields) are denoted, respectively, by $\widetilde{u}_{i}$ and $\widetilde{\Theta}$. The tildes are used to distinguish the conditional average from the standard (long-time, ensemble, or plane) average $\bar{X}$, which is a function of $z$ only. The symmetry-accounted average can be interpreted exactly as classical Reynoldsaveraged results. For further details we refer to the accompanying paper [9].

\section{RESULTS}

\section{A. Boundary layer thickness}

The thicknesses of the hydrodynamic and thermal boundary layers as a function of Ra are shown in Fig. 1. Here, $\lambda_{u}$ and $\lambda_{\Theta}$ are defined as the location of the maximum of the mean squared horizontal velocity fluctuations $\overline{u^{\prime} u^{\prime}}$ and mean squared temperature fluctuations $\overline{\Theta^{\prime} \Theta^{\prime}}$, respectively. The approximate power laws are $\lambda_{u}=0.5 \mathrm{Ra}^{-0.13}$ and $\lambda_{\Theta}$ $=2.33 \mathrm{Ra}^{-0.27}$, respectively, in good agreement with other simulations [4] and reasonable agreement with experiments [2] (despite differences in aspect ratio, geometry, and boundary conditions).

Also shown in Fig. 1 are the predictions of the boundary layer thickness (1) and (2) from the Grossmann-Lohse (G-L) theory [1], together with the DNS results. The thermal boundary layer thickness $\lambda_{\Theta}$ is in good agreement with the simulations. The width of the kinematic boundary layer $\lambda_{u}$ does not agree so well with the G-L theory, as the actual exponent is -0.13 instead of -0.25 (where we have assumed free-fall scaling $\operatorname{Re} \propto \mathrm{Ra}^{1 / 2}$ for simplicity).

Below we briefly recapitulate the arguments of [7] leading to (2). The starting point is the laminar two-dimensional Prandtl boundary layer equation $[6,15]$

$$
u \partial_{x} u+w \partial_{z} u=\nu \partial_{z}^{2} u .
$$

Upon substituting $x \rightarrow H x, z \rightarrow H \operatorname{Re}^{-1 / 2} z, u \rightarrow U u$, and $w$ $\rightarrow U \operatorname{Re}^{-1 / 2} w$, the equations become parameter independent as

$$
u \partial_{x} u+w \partial_{z} u=\partial_{z}^{2} u .
$$

Neither this expression, nor the incompressibility conditions, nor the boundary conditions have an explicit dependence on 


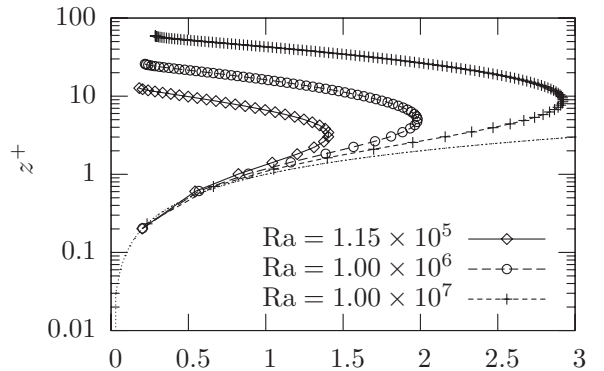

(a)

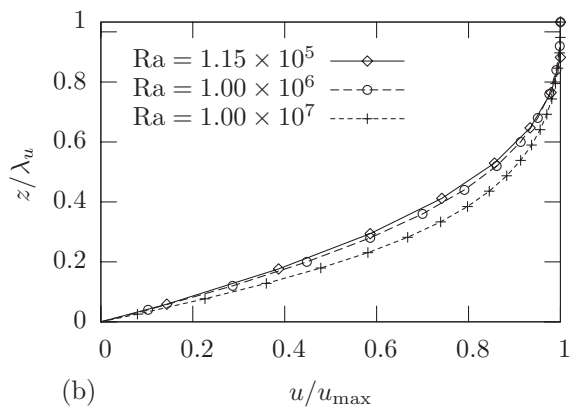

FIG. 2. Horizontal boundary layer velocity profile based on $\sqrt{\langle\widetilde{u} \widetilde{u}\rangle_{A}}$ for various Ra. (a) Semilogarithmic plot, nondimensionalized with friction velocity $u_{\tau}$. Dashed line represents $u^{+}=z^{+}$; (b) normalized by the maximum velocity $u_{\max }$ and the kinematic boundary layer thickness $\lambda_{u}$.

$\mathrm{Re}$, so the solution has to be independent of $\mathrm{Re}$ as well. Therefore, the flow pattern undergoes a similarity transformation, and the boundary layer thickness scales as $\lambda_{u} / H$ $\propto \mathrm{Re}^{-1 / 2}$. This result is rigorous, provided that (3) holds, i.e., that turbulent stresses do not play a role in the momentum budget. In Sec. III E we show that forcing due to Reynolds stresses cannot be neglected for the boundary layer equations so that the laminarity assumption does not hold.

\section{B. Velocity profiles}

The characteristic shape of the velocity profile can be obtained from the plane-averaged horizontal average velocity as $u(z) \approx \sqrt{\langle\tilde{u} \tilde{u}\rangle_{A}}$. Figure 2(a) shows these profiles for various $\mathrm{Ra}$ in plus units, i.e., scaled by the friction velocity $u_{\tau} \equiv \sqrt{\tau_{w} / \rho}$ with $u^{+}=u / u_{\tau}$ and $z^{+}=z u_{\tau} / \nu$. Here, we define a typical wall shear stress $\tau_{w}$ as

$$
\tau_{w}=\left.\rho \nu \partial_{z} \sqrt{\langle\tilde{u} \tilde{u}\rangle_{A}}\right|_{w} .
$$

In Fig. 2(a), the viscous sublayer relation $u^{+}=z^{+}$is shown with a dashed line, and logarithmic scaling of the velocity profile results in a straight line. For a classical turbulent channel flow and constant-pressure boundary layer, the viscous region ends at $y^{+}=5$, the logarithmic layer starts from $y^{+} \approx 30$, and the profiles will collapse onto a single universal curve for all Re. Here the situation is completely different. First, in plus coordinates the profiles do not collapse onto a single curve. Furthermore, the viscous region ends at approximately $z^{+}=1$, and the velocity reaches its maximum at $z^{+} \approx 10$ at $\mathrm{Ra}=10^{7}$. A region where the velocity scales loga-
TABLE I. Characteristic numbers for the boundary layer profile at various Ra: the shear Reynolds number $\operatorname{Re}_{\tau}$, the shape factor $S$, and the friction coefficient $C_{f}$.

\begin{tabular}{cccc}
\hline \hline $\mathrm{Ra}$ & $\mathrm{Re}_{\tau}$ & $C_{f}$ & $S$ \\
\hline $1.15 \times 10^{5}$ & 26 & 1.02 & 2.37 \\
$1.00 \times 10^{6}$ & 52 & 0.51 & 2.35 \\
$1.00 \times 10^{7}$ & 119 & 0.23 & 2.27 \\
\hline \hline
\end{tabular}

rithmically cannot be distinguished, indicating the absence of an inner (constant-stress) layer.

Shown in Fig. 2(b) is the velocity profile normalized by the outer variables, i.e., the boundary layer thickness $\lambda_{u}$ and the maximum velocity $u_{\max } \equiv u\left(\lambda_{u}\right)$. Although the profiles show that there is a $\mathrm{Ra}$ dependence, it is very weak. The weak influence of the $\mathrm{Ra}$ number-especially for the two lower Ra numbers considered-is further evidence that the kinematic boundary layer does not behave as a classical forced turbulent boundary layer. Note that the approximate universality of the velocity profiles means that inner and outer variables can be interchanged, in the sense that $\left.\partial_{z} u\right|_{w}$ $\propto u_{\text {max }} / \lambda_{u}$.

Several experiments have shown universality in Ra upon an outer scaling by boundary layer thickness and maximum velocity $[2,16,17]$, so it is quite interesting that the boundary layer profile found here [Fig. 2(b)] has a (small) Ra dependence. There may be several reasons for this difference. The experiments have been carried out at much higher $\mathrm{Ra}$, in the range $\mathrm{Ra}=2 \times 10^{8}-9 \times 10^{9}$, and at higher $\operatorname{Pr}$ (the working fluid was water). Furthermore, the presence of sidewalls and the smaller aspect ratio will be of influence.

It is useful to express the shear Reynolds number in terms of $\mathrm{Re}, \lambda_{u}$, and the nondimensional velocity gradient at the wall. Let the outer scaled variables be denoted by $\hat{z} \equiv z / \lambda_{u}$ and $\hat{u} \equiv u / u_{\max }$. The nondimensional velocity gradient at the wall is connected to the wall-shear stress by $\tau_{w}$ $=\left.\rho \nu u_{\max } \lambda_{u}^{-1} \partial_{\hat{z}} \hat{u}\right|_{w}$, where $\left.\partial_{\hat{z}} \hat{u}\right|_{w}$ is the nondimensional velocity gradient at the wall. Hence, the shear Reynolds number can be expressed as

$$
\operatorname{Re}_{\tau}=\operatorname{Re}^{1 / 2}\left(\frac{\lambda_{u}}{H}\right)^{-1 / 2}\left(\left.\partial_{\hat{z}} \hat{u}\right|_{w}\right)^{1 / 2}
$$

All three terms $\operatorname{Re}, \lambda_{u}$, and $\left.\partial_{\hat{z}} \hat{u}\right|_{w}$ depend on Ra, although the $\mathrm{Ra}$ dependence of the last term is very weak as $\left.\partial_{\hat{z}} \hat{u}\right|_{w}$ $\propto \mathrm{Ra}^{0.06}$.

\section{Friction and shape factors}

The friction and shape factors [e.g., [6,15]] have been calculated for all three $\mathrm{Ra}$ (Table I). The friction factor is defined as

$$
C_{f}=\frac{\tau_{w}}{\frac{1}{2} u_{\max }^{2}}=2 \frac{\mathrm{Re}_{\tau}^{2}}{\mathrm{Re}^{2}} .
$$

Here we note that combining (6) and (7) and neglecting the small Ra dependence of the wall gradient $\left.\partial_{\hat{z}} \hat{u}\right|_{w}$ gives that $C_{f}$ can be approximated by 


$$
C_{f} \approx \frac{2}{\operatorname{Re}}\left(\frac{\lambda_{u}}{H}\right)^{-1} .
$$

This is consistent with the approximation $\tau_{w} \approx \nu U / \lambda_{u}$, which is an important assumption in the Grossmann-Lohse theory [1]. The observation (8) will prove to be important to establish the scaling of $\lambda_{u} / H$ in Sec. IV.

Based on the values of Table I and in terms of Re, the friction factor $C_{f}$ scales as $C_{f} \propto \mathrm{Re}^{-0.60}$. An empirical relation for turbulent plane channel flow is $C_{f}=0.073 \mathrm{Re}^{-0.25}$, with $\mathrm{Re}$ based on the channel half-width and mean velocity across the channel [18]. The friction factor of laminar boundary layers has a stronger dependence on Re; for plane Poiseuille flow $C_{f}=8 / \operatorname{Re}(\operatorname{Re}$ based on full channel height) and for the Blasius flat plate flow $C_{f}=0.664 \mathrm{Re}_{x}^{-1 / 2}$. Hence, judging from the scaling of the friction factor, the behavior of the boundary layer would be classified as laminar. These results are consistent with [10-12].

The shape factor $S$ is defined as $S=\delta_{1} / \delta_{2}$, where $\delta_{1}$ and $\delta_{2}$ are the displacement and momentum thickness, given by

$$
\begin{gathered}
\delta_{1}=\int_{0}^{\lambda_{u}}\left(1-\frac{u}{u_{\max }}\right) d z, \\
\delta_{2}=\int_{0}^{\lambda_{u}} \frac{u}{u_{\max }}\left(1-\frac{u}{u_{\max }}\right) d z .
\end{gathered}
$$

For laminar profiles, such as Poiseuille flow and the Blasius solution for the developing flow over a flat plate, the shape factor is approximately 2.5 (e.g., $[6,15]$ ). For turbulent plane channel flow, flat-plate constant-pressure boundary layers, and a plane turbulent wall jet [19], the shape factor is approximately 1.3-1.4. Based on this information, the values from Table I indicate that the velocity profile follows a laminarlike distribution with a slight trend toward turbulent values as Ra increases.

If the shape and friction factors were taken to be representative to distinguish a laminar from a turbulent boundary layer, the boundary layer would be classified as laminar. In the next sections we will study the momentum budgets of the boundary layers, and compare the time and space spectra of the boundary layer and the bulk. It will be shown that, from this perspective, the kinematic boundary layer has many features of turbulence.

\section{Fluctuations and spectra}

In Figs. 3(a)-3(c) the average velocity profile $\langle\tilde{u}\rangle_{y} / u_{\max }$ is shown for $\mathrm{Ra}=1.15 \times 10^{5}, 10^{6}$, and $10^{7}$, together with the turbulence intensity of the horizontal and vertical fluctuations, $\left\langle u^{\prime} u^{\prime}\right\rangle_{y}^{1 / 2} / u_{\max }$ and $\left\langle w^{\prime} w^{\prime}\right\rangle_{y}^{1 / 2} / u_{\max }$, respectively. These are the profiles of the $y$-averaged wind structure (see Fig. 4), with the $x$ location chosen such that the horizontal velocity is at its maximum, i.e., where the flow is parallel to the wall and from left to right. A striking feature of the turbulence intensity of the horizontal fluctuations is that it is so large compared to the mean wind, namely, 70-80\%. For turbulent channel flow, typical turbulence intensities are 5-10\%. Outside the thermal boundary layer the horizontal turbulence

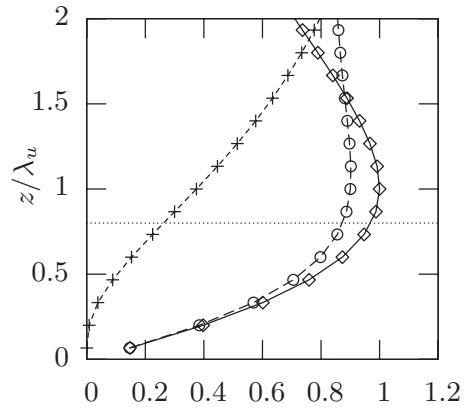

(a) $u / u_{\max }$

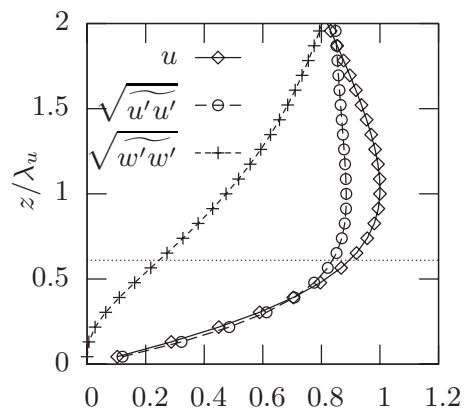

(b) $u / u_{\max }$

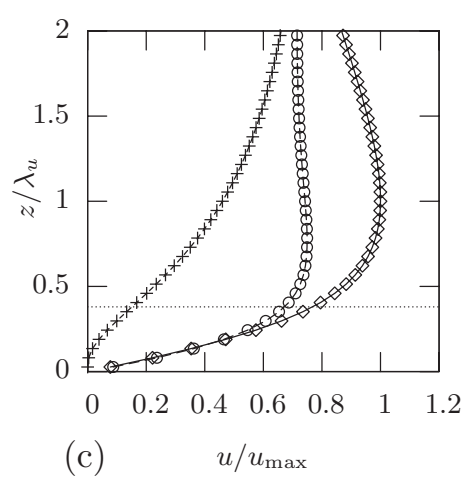

FIG. 3. Closeup of horizontal velocity and turbulence intensities [legend in (b)] at the position with the maximum horizontal velocity. $\mathrm{Ra}=$ (a) $1.15 \times 10^{5}$, (b) $1.00 \times 10^{6}$, and (c) $1.00 \times 10^{7}$. The horizontal dashed line indicates the edge of the thermal boundary layer $\lambda_{\Theta}$.

intensity is constant. The vertical turbulence intensity is not as large as the horizontal due to wall blocking, but is still $20 \%$ at the edge of the thermal boundary layer, and $50 \%$ at the edge of the kinematic boundary layer. This confirms that fluctuations in large-aspect-ratio domains are larger relative to the wind [20], in comparison with those in small-aspectratio domains (e.g., [2] reports turbulence intensities of $20 \%)$.

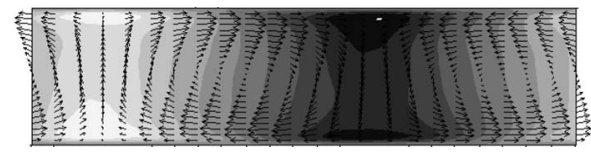

FIG. 4. $y$-averaged wind structure at $\operatorname{Ra}=10^{6}$ and $\operatorname{Pr}=1$. Color scheme is by the relative temperature $\Theta_{r}=\widetilde{\Theta}-\langle\widetilde{\Theta}\rangle_{A}$. Dark areas are relatively cold, and white areas are relatively hot. 

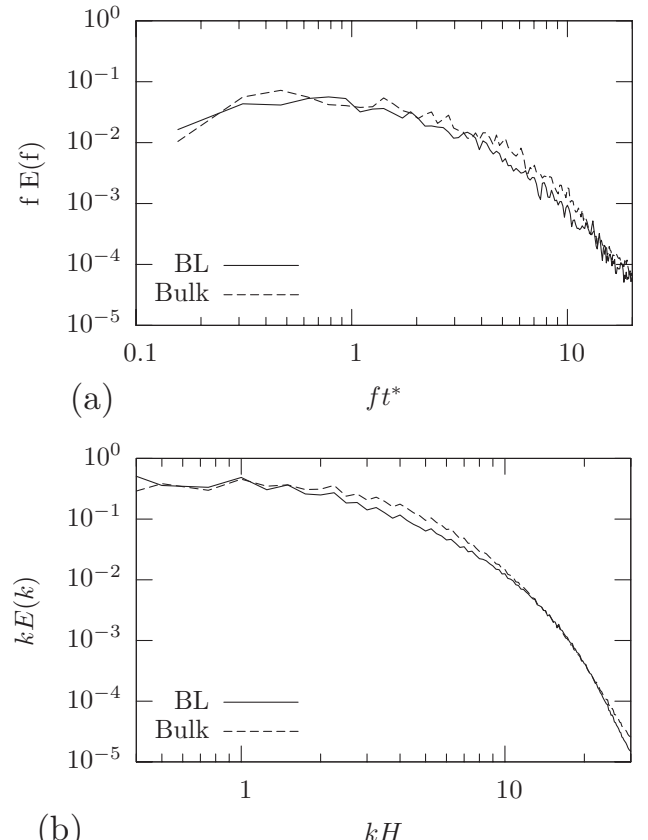

FIG. 5. Spectra of the horizontal velocity components in the boundary layer and in the bulk at $\mathrm{Ra}=10^{7}$ normalized by the bulk variance: (a) temporal and (b) spatial spectrum.

One of the main features of turbulence is the presence of a continuous range of active scales. A simulation at $\mathrm{Ra}$ $=10^{7}$ is used to obtain both spatial and temporal spectra of the horizontal velocity components. To collect temporal spectra, eight points have been monitored: four bulk and four boundary layer points. The bulk points are taken at $z_{\text {bulk }}$ $=H / 2$ and the boundary layer points were chosen according to $z_{\mathrm{bl}}=\lambda_{u}$. The temporal spectra are generated by segmenting the time series and a Welch window has been used. Then, averaging was performed over the spectra of the two horizontal velocity components and the four monitoring points. The spatial spectra were collected by performing a two dimensional fast Fourier transform (FFT), integrating over circles $k_{x}^{2}+k_{y}^{2}=k^{2}$, and averaging over approximately ten turnovers.

The temporal spectra of the horizontal velocity components at $\mathrm{Ra}=10^{7}$ are shown in Fig. 5(a). There is a continuous range of active scales which spans about two decades, although turbulence production and dissipation are not sufficiently separated to form a clearly discernible inertial subrange. The spatial spectra [Fig. 5(b)] also reveal a continuous range of active scales.

What is striking about the spectra of the bulk and the boundary layer is how similar they are, both in range of active scales and in amplitude. Despite a mild damping at the intermediate frequencies and wave numbers, the similarity indicates that the dynamics of the bulk and the boundary layer-both temporal and spatial - are very similar. We note that the simulation at $\mathrm{Ra}=10^{7}$ is well inside the hardturbulence regime. The transition to hard turbulence occurs at much lower $\mathrm{Ra}$ for large-aspect-ratio domains than the generally accepted value of $\mathrm{Ra}=4 \times 10^{7}$ [21]. Indeed, for aspect-ratio- 6 domains, the flow has hard-turbulence regime scaling from $\mathrm{Ra}=5 \times 10^{4}$ upwards [4]. If one accepts that the flow core is turbulent, then Fig. 5 indicates that the boundary layers are turbulent as well.

The striking similarity between the spectra in the bulk and the boundary layers seems to be a robust and general feature of Rayleigh-Bénard convection. In a recent paper [13], we present combined experimental and numerical results of an aspect-ratio-4 cavity filled with water for Rayleigh numbers ranging from $5 \times 10^{4}$ to $10^{9}$. For all $\mathrm{Ra}$ from $10^{6}$ upward, it is found that the spectra in the bulk and the boundary layer are practically identical.

\section{E. Momentum budgets}

Momentum budgets are a very direct way to get an impression of the importance of the turbulent Reynolds stresses. As before, $y$-averaged results (Fig. 4) are used for convenience of presentation. Checks have been made to ensure that the budgets shown here are also representative for the three-dimensional field. The $x$ location has been chosen such that the horizontal velocity is at its maximum, i.e., where the flow is parallel to the wall and from left to right. This guarantees that horizontal gradients are small, and that no adverse or favorable pressure gradients are present. Shown are budgets for $\mathrm{Ra}=1.15 \times 10^{5}$ [Figs. 6(a), 6(d), and $6(\mathrm{~g})], 1.00 \times 10^{6}$ [Figs. 6(b), 6(e), and 6(h)], and $1.00 \times 10^{7}$ [Figs. 6(c), 6(f), and 6(i)]. The budgets for the horizontal [Figs. 6(a)-6(c))] and vertical momentum [Figs. 6(d)-6(f))] have been nondimensionalized by $U^{2} / H=\beta g \Delta \Theta$, and the heat budget [Figs. $6(\mathrm{~g})-6(\mathrm{i})]$ by $\Delta \Theta U / H=\sqrt{\beta g(\Delta \Theta)^{3} / H}$. The legend for the budgets is shown in Fig. 6(e) and the budget terms are defined in Table II. The $z$ coordinate has been scaled by $\lambda_{u}$ and the horizontal dashed line denotes $z$ $=\lambda_{\Theta}$. For reference, the ratio $\lambda_{\Theta} / \lambda_{u}$ is $0.8,0.6$, and 0.38 for the simulations at $\mathrm{Ra}=10^{5}, 10^{6}$, and $10^{7}$, respectively.

For the horizontal momentum budgets [Figs. 6(a)-6(c)], the balance is between the horizontal pressure gradient $\mathcal{P}$ and diffusion $\mathcal{D}$ for $z<\lambda_{\Theta}$. Outside the thermal boundary layer, $\mathcal{R}$ is not negligible; on the contrary, $\mathcal{R}$ fully balances the pressure gradient $\mathcal{P}$ near $z=\lambda_{u}$. This indicates that the turbulence outside the thermal boundary layer is key to the boundary layer thickness, as will be outlined in Sec. VI. As the location of the budgets has been chosen such that all horizontal derivatives are small, $\mathcal{D} \approx \nu \partial_{z}^{2} \tilde{u}$ and $\mathcal{R} \approx-\partial_{z} \widetilde{w^{\prime} u^{\prime}}$.

Logarithmic scaling is expected in the inner layer where $\widetilde{w^{\prime} u^{\prime}}$ is constant, so that $\mathcal{R}=-\partial_{z} \widetilde{w^{\prime} u^{\prime}}=0$. For channel flow, $\mathcal{R}$ is zero at the wall and peaks in the buffer layer, marking the transport of momentum from the outer to the inner layer. After the peak, it crosses the zero axis where the logarithmic layer is expected. This behavior of $\mathcal{R}$ is absent for $\mathrm{Ra}$ $=1.15 \times 10^{5}$, but as $\mathrm{Ra}$ increases a peak forms inside the thermal boundary layer [Figs. 6(a)-6(c)]. However, in terms of forcing, $\mathcal{P}$ is always much larger than the small peak $\mathcal{R}$ for the range of $\mathrm{Ra}$ under consideration, which again confirms that this is not a classical forced turbulent boundary layer.

Figures $6(\mathrm{~d})-6(\mathrm{f})$ show the budgets of the $\widetilde{w}$ momentum equation. Here the balance is between buoyancy $\mathcal{B}$, the vertical pressure gradient $\mathcal{P}$, and the Reynolds stresses $\mathcal{R}=$ 


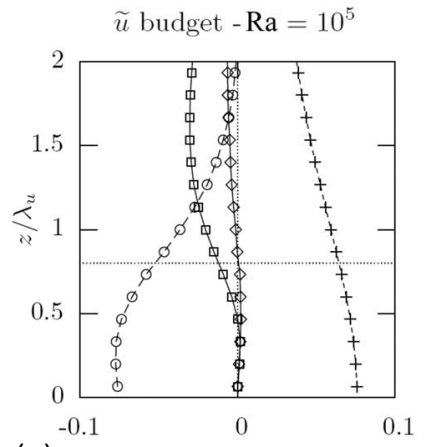

(a)

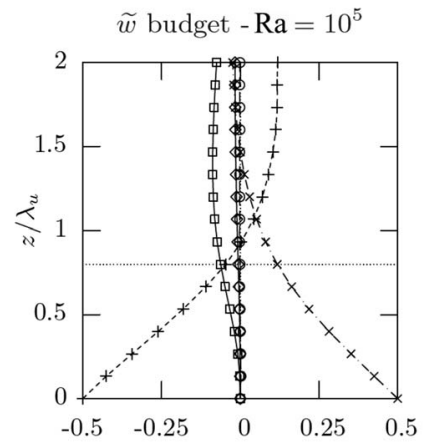

(d)

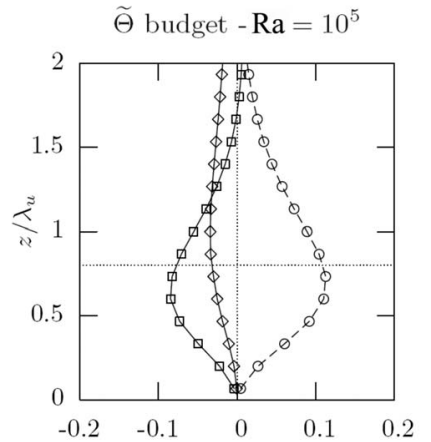

(g)

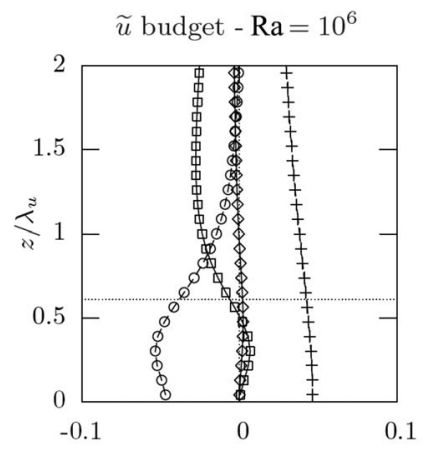

(b)

$$
\widetilde{w} \text { budget }-\mathrm{Ra}=10^{6}
$$

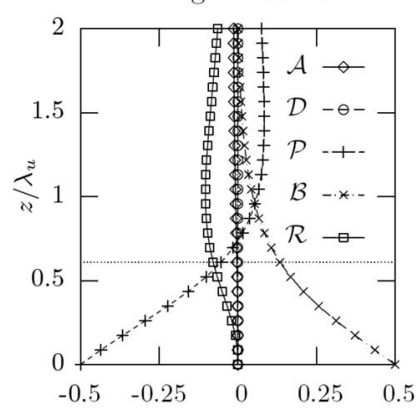

(e)

$$
\widetilde{\Theta} \text { budget }-\mathrm{Ra}=10^{6}
$$

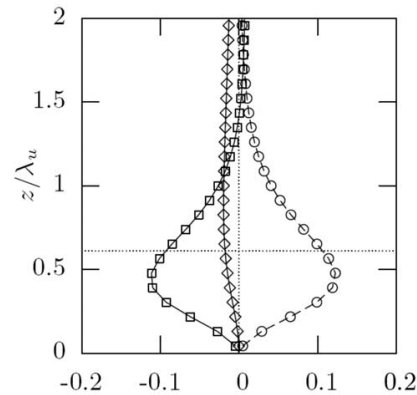

(h)

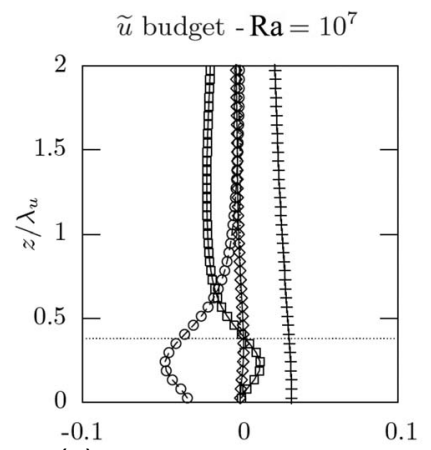

(c)

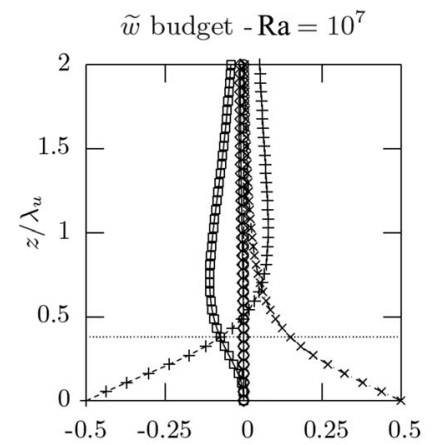

(f)

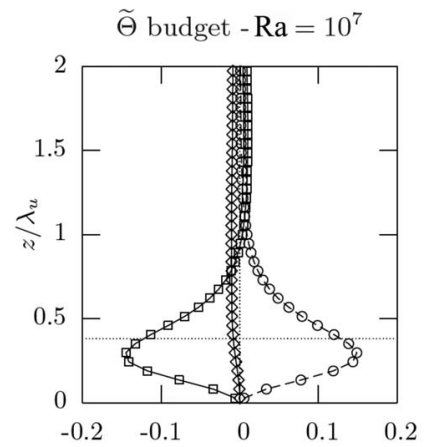

(i)

FIG. 6. Momentum and heat budgets in the boundary layer at the position with the maximum horizontal velocity for various Ra: Ra $=(\mathrm{a}),(\mathrm{d}),(\mathrm{g}) 1.15 \times 10^{5}$; (b), (e), (h) $1.00 \times 10^{6}$; (c),(f),(i) $\mathrm{Ra}=1.00 \times 10^{7}$. (a),(b),(c) $\tilde{u}$ and (d), (e), (f) $\tilde{w}$ momentum budget; (g), (h), (i) $\widetilde{\Theta}$ budget. The legend for (a)-(i) is shown in (e) and the horizontal dashed line indicates the edge of the thermal boundary layer $z=\lambda_{\Theta}$.

$-\partial_{z} \widetilde{w^{\prime} w^{\prime}}$. Very near the wall, roughly in the lower half of the thermal boundary layer, the buoyancy $\mathcal{B}$ and pressure $\mathcal{P}$ are in balance, so the flow is neutrally buoyant here. Further away from the wall, at the edge of the thermal boundary layer, the contribution of $\mathcal{R}$ is significant, even if it may seem small compared to the near-wall (hydrostatic) balance of $\mathcal{P}$ and $\mathcal{B}$. In fact, comparing $\mathcal{R}$ of the vertical momentum equation to the magnitude of terms in the horizontal momentum equation shows that it is of the same magnitude as $-\partial_{x} \tilde{p}$. Outside the boundary layer, the pressure gradient $\mathcal{P}$ is posi-

TABLE II. Budget terms for momentum and heat equation.

\begin{tabular}{cccccc}
\hline \hline & $\mathcal{A}$ & $\mathcal{D}$ & $\mathcal{P}$ & $\mathcal{B}$ & $\mathcal{R}$ \\
\hline$\partial_{t} \tilde{u}_{i}=$ & $-\partial_{j} \tilde{u}_{j} \widetilde{u}_{i}$ & $+\nu \partial_{j}^{2} \tilde{u}_{i}$ & $-\partial_{i} \tilde{p}$ & $+\beta g \widetilde{\Theta} \delta_{i 3}$ & $-\partial_{j} \widetilde{u_{j}^{\prime} u_{i}^{\prime}}$ \\
$\partial_{t} \widetilde{\Theta}=$ & $-\partial_{j} \widetilde{u}_{j} \widetilde{\Theta}$ & $+\kappa \partial_{j}^{2} \widetilde{\Theta}$ & & & $-\partial_{j} \tilde{u}_{j}^{\prime} \Theta^{\prime}$ \\
\hline \hline
\end{tabular}

tive and is balanced purely by fluctuations $\mathcal{R}$.

The $\widetilde{\Theta}$ momentum budgets [Figs. 6(h) and 6(i)] show a balance between thermal diffusion $\mathcal{D}=\kappa \partial_{z}^{2} \widetilde{\Theta}$, turbulence $\mathcal{R}$ $=-\partial_{z} \widetilde{w^{\prime} \Theta^{\prime}}$, and a contribution from advection $\mathcal{A}$. Judging from the peak of $\mathcal{A}$ around $z / \lambda_{u}=1$, the nonzero contribution of $\mathcal{A}$ to the heat budget is probably caused by some spatial variations in $\widetilde{\Theta}$ by which $\partial_{x} \tilde{u} \widetilde{\Theta} \neq 0$. The peak of $\mathcal{D}$ and $\mathcal{R}$ is always located just inside the thermal boundary layer, representing the location where diffusion and fluctuations most effectively exchange heat.

It is striking that the dominant length scale for the budgets is the thermal boundary layer thickness $\lambda_{\Theta}$ (which is denoted by the horizontal dashed line in Fig. 6), and not, as one may expect, the kinematic boundary layer thickness. Perhaps this should not be too much of a surprise, as the thermal boundary layer thickness can be well represented by $\lambda_{\Theta}$ $=H /(2 \mathrm{Nu})$, and the Nusselt number $\mathrm{Nu}$ represents the effi- 
ciency of the convective heat transfer mechanism of the flow, resulting from the nonlinear coupling of temperature and velocity under the action of buoyancy. Therefore, $\lambda_{\Theta}$ is equally important for the heat budget and for the momentum budgets. In fact, $\lambda_{\Theta}$ is a dominant parameter in the scaling of both $C_{f}$ and $\lambda_{u}$, as will be shown in Sec. IV.

The findings of Figs. 6(a)-6(i) can be summarized as follows for the $\widetilde{u}, \widetilde{w}$, and $\widetilde{\Theta}$ budgets, respectively:

$$
\begin{gathered}
\partial_{x} \widetilde{p}+\partial_{z} \widetilde{w^{\prime} u^{\prime}}=\nu \partial_{z}^{2} \widetilde{u}, \\
\partial_{z} \widetilde{p}+\partial_{z} \widetilde{w^{\prime} w^{\prime}}=\beta g \widetilde{\Theta}, \\
\partial_{z} \widetilde{w^{\prime} \Theta^{\prime}}=\kappa \partial_{z}^{2} \widetilde{\Theta} .
\end{gathered}
$$

These equations represent the boundary layer equations at the $x$ location where the flow is parallel to the wall and horizontal derivatives are negligible (roughly halfway between the impingement and detachment region). Note that, even though the $\widetilde{w}$ momentum equation is not directly coupled to the other two equations, the vertical fluctuations $\widetilde{w^{\prime} w^{\prime}}$ are nontrivially coupled to $\widetilde{w^{\prime} u^{\prime}}$ and $\widetilde{w^{\prime} \Theta^{\prime}}$, as these terms represent to a large extent the plumes emerging from and impinging on the boundary layers. The equations above are two dimensional, but from the absence of transversal derivatives, it can be expected that these equations are valid for the three-dimensional case as well, in a local coordinate system aligned with the flow and at the location where the flow is parallel to the wall.

The boundary layer equation (9) clearly shows that one cannot neglect the influence of turbulence in the boundary layer dynamics. Hence, the laminar boundary layer equation (3), which leads to the scaling $\lambda_{u} / H \propto \mathrm{Re}^{-1 / 2}$, is not valid: additional information is required about ${w^{\prime} u^{\prime}}^{\prime}$ to estimate $\lambda_{u}$. In Sec. IV, the scaling behavior of $\lambda_{u}$ will be derived using flow-specific information obtained from the DNS results.

\section{F. The friction factor decomposed}

By using the boundary layer equation (9), the dominant contributor to the friction factor can be identified. Integrating (9) over the kinematic boundary layer and substituting (7), the friction factor $C_{f}$ is composed of a contribution from pressure and a turbulent momentum flux as

$$
\frac{C_{f}}{2}=\frac{1}{u_{\max }^{2}} \int_{0}^{\lambda_{u}}(\mathcal{P}+\mathcal{R}) d z=-\frac{1}{u_{\max }^{2}} \int_{0}^{\lambda_{u}} \partial_{x} p d z-\frac{\left.\widetilde{w^{\prime} u^{\prime}}\right|_{\lambda_{u}}}{u_{\max }^{2}} .
$$

The terms on the right-hand side of (12) have been calculated with the DNS results and are presented in Table III [25]. The decomposition clearly demonstrates that $C_{f}$ is dominated by the pressure gradient. The turbulent momentum flux $\widetilde{w^{\prime} u^{\prime}}$ is small but positive, i.e., a flux out of the boundary layer. Hence, we conclude that the dynamics of the wall friction is not governed by turbulence (i.e., Reynolds stress) as in a forced turbulent boundary layer. In the latter, $C_{f}$ is dominated by a large momentum flux into the boundary
TABLE III. Decomposition of the friction factor $C_{f}$ according to (12).

\begin{tabular}{cccc}
\hline \hline $\mathrm{Ra}$ & $C_{f}=$ & $-\left(2 / u_{\max }^{2}\right) \int_{0}^{\lambda_{u}} \partial_{x} p d z$ & $-\left.2 \widetilde{w^{\prime} u^{\prime}}\right|_{\lambda_{u}} / u_{\max }^{2}$ \\
\hline $10^{5}$ & 0.81 & 0.88 & -0.07 \\
$10^{6}$ & 0.39 & 0.42 & -0.03 \\
$10^{7}$ & 0.17 & 0.19 & -0.02 \\
\hline \hline
\end{tabular}

layer, while the contribution of the pressure gradient is negligible.

Using (12), $C_{f}$ can be parametrized. Shown in Fig. 7 is the effective forcing $\mathcal{P}+\mathcal{R}=-\partial_{x} \widetilde{p}-\partial_{z} \widetilde{w^{\prime} u^{\prime}}$. To first order, for $z$ $<\lambda_{\Theta}, \mathcal{P}+\mathcal{R} \approx \mathcal{P}$ while for $z>\lambda_{\Theta}$ the Reynolds stress forcing $\mathcal{R}$ balances the pressure $\mathcal{P}$ to that $\mathcal{P}+\mathcal{R} \approx 0$. Hence, $C_{f}$ can be estimated via

$$
C_{f} \approx \frac{1}{u_{\max }^{2}} \int_{0}^{\lambda_{\Theta}} \mathcal{P} d z \approx \frac{2 \lambda_{\Theta}}{u_{\max }^{2}}\left|\partial_{x} p\right|_{w} \mid .
$$

Clearly, (13) holds at moderate Ra only, when turbulent shear production in the boundary layer is small. The formation of the peak inside the thermal boundary layer at Ra $=10^{6}$ and $10^{7}$ (Fig. 7) suggests that shear production becomes more important as Ra increases, and this will have to be accounted for in (13) at higher Ra. However, it was shown in the accompanying paper [9] that the wind velocity becomes independent of $C_{f}$ at sufficiently high Ra, because $C_{f}$ is negligible compared to the mixing parameter $\alpha$. Therefore, incorrect scaling behavior in (13) will not influence the wind dynamics at high Ra.

\section{SCALING OF $C_{f}$ AND $\lambda_{u}$}

Using the simple two-equation wind model derived in the accompanying paper [9], we can establish the scaling behavior of $C_{f}$ and $\lambda_{u}$. The model uses a dimensionless wind velocity $\hat{U}_{w}=U_{w} / U_{f}$ and spatial temperature difference $\hat{\Theta}_{w}$ $=\Theta_{w} / \Delta \Theta$, where $U_{f}=\sqrt{\beta g \Delta \Theta H}$ is the free-fall velocity. The governing equations of the model are given by

$$
\frac{d \hat{U}_{w}}{d \hat{t}}=\frac{2 \hat{L}_{w}^{2}}{2 \hat{L}_{w}^{2}+1}\left(\frac{1}{2 \hat{L}_{w}} \hat{\Theta}_{w}-\left(4 \alpha+C_{f}\right)\left|\hat{U}_{w}\right| \hat{U}_{w}\right),
$$

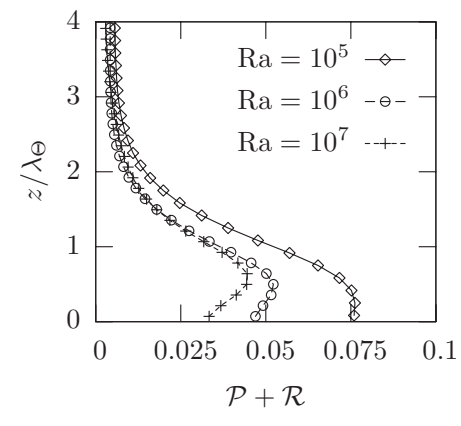

FIG. 7. Effective forcing $\mathcal{P}+\mathcal{R}=-\partial_{x} \widetilde{p}-\partial_{z} \widetilde{w^{\prime} u^{\prime}}$, which vanishes quickly outside the thermal boundary layer. 


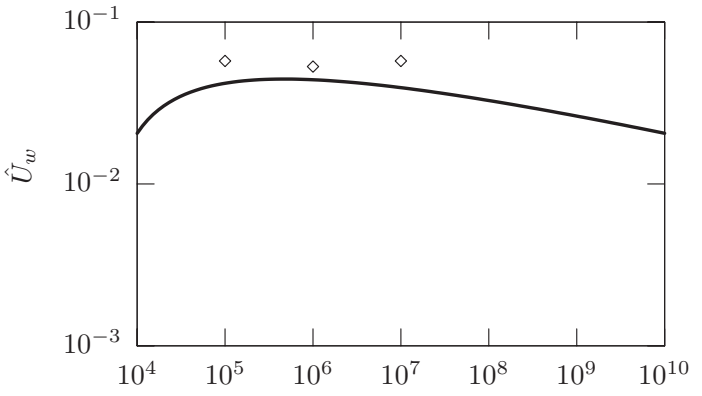

(a)

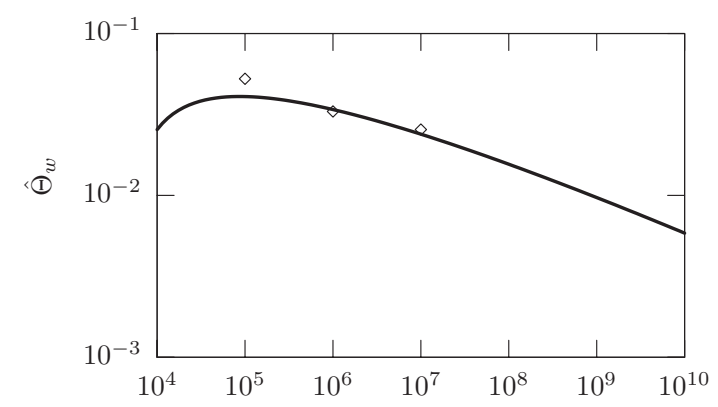

(b)
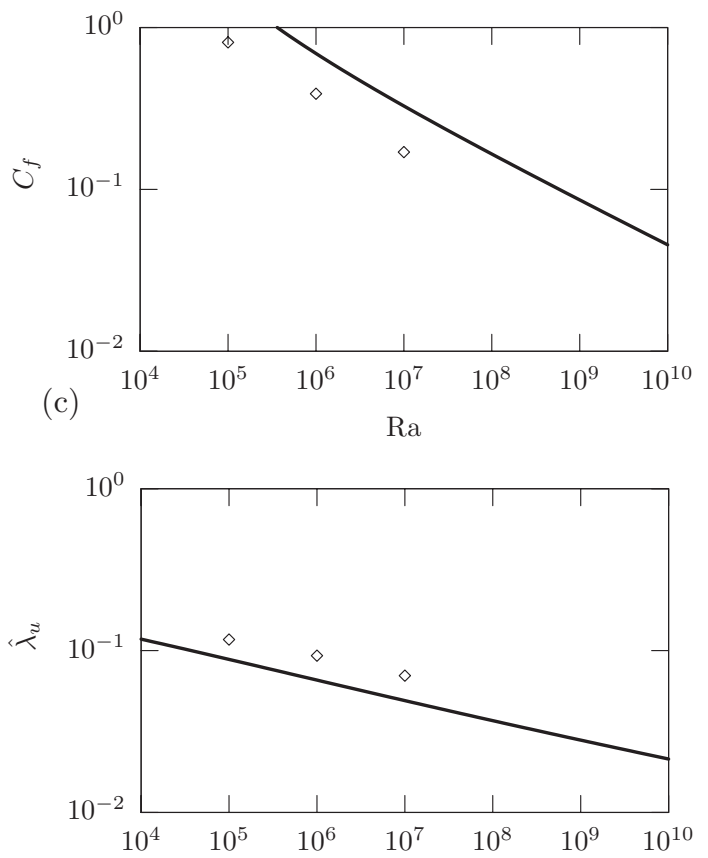

(d)

$\mathrm{Ra}$

FIG. 8. Predictions of the wind model [Eqs. (19) and (20), thick lines] compared to the DNS results (diamonds) for (a) the typical wind $\hat{U}_{w}$, (b) the spatial temperature difference $\hat{\Theta}_{w}$, (c) the friction factor $C_{f}$, and (d) the kinematic boundary layer thickness $\lambda_{u}$.

$$
\frac{d \hat{\Theta}_{w}}{d \hat{t}}=\frac{2 \hat{\lambda}_{\Theta}}{\hat{L}_{w}} \hat{U}_{w}-\frac{4 \alpha}{\hat{L}_{w}^{2} \operatorname{Pr}_{T}}\left|\hat{U}_{w}\right| \hat{\Theta}_{w}-\frac{2}{\hat{\lambda}_{\Theta} \operatorname{Re}_{f} \operatorname{Pr}} \hat{\Theta}_{w} .
$$

Here $\hat{L}_{w}=L_{w} / H$ where $L_{w}$ is the typical roll size, $\hat{\lambda}_{\Theta}=\lambda_{\Theta} / H$, $\hat{\lambda}_{u}=\lambda_{u} / H$, and $\operatorname{Re}_{f}=U_{f} H / \nu=\operatorname{Ra}^{1 / 2} \operatorname{Pr}^{-1 / 2}$. The turbulent Prandtl number $\operatorname{Pr}_{T}$ and the mixing parameter $\alpha$ are coeffi- cients with values 0.85 [6] and 0.6 , respectively. The pressure difference which drives the wind is generated by a spatial temperature difference $\Theta_{w}$ (it is relatively hot where the flow ascends and relatively cold where it descends; see Fig. 4). The temperature difference $\Theta_{w}$ is in its turn generated by large horizontal heat fluxes originating from the interaction between the mean wind and temperature field. The model depends on $\mathrm{Ra}$, Pr, and $\hat{L}_{w}$, where $\hat{\lambda}_{\Theta}=\hat{\lambda}_{\Theta}(\operatorname{Ra}, \operatorname{Pr})$ and $C_{f}$ $=C_{f}(\mathrm{Ra}, \mathrm{Pr})$ have to be provided. Based on the analysis of the friction factor (Sec. III F), an explicit expression for $C_{f}$ can be derived, by which the model depends only on empirical input for $\hat{\lambda}_{\Theta}$ (and thus $\mathrm{Nu}$ ).

The steady state estimate for the pressure gradient at the bottom wall of the wind model is [9]

$$
-\left.\partial_{x} \tilde{p}\right|_{w} \approx \frac{\beta g H}{L_{w}} \Theta_{w}
$$

Using (16), $C_{f}(13)$ can be further specified as

$$
C_{f} \approx \frac{2 \lambda_{\Theta}}{H} \frac{H}{L_{w}} \frac{U_{f}^{2}}{U_{w}^{2}} \frac{\left|\Theta_{w}\right|}{\Delta \Theta}=\frac{2 \hat{\lambda}_{\Theta}\left|\hat{\Theta}_{w}\right|}{\hat{L}_{w} \hat{U}_{w}^{2}} .
$$

Hence, the wall friction term is linear in the temperature difference,

$$
C_{f}\left|\hat{U}_{w}\right| \hat{U}_{w}=\frac{2 \hat{\lambda}_{\Theta}}{\hat{L}_{w}} \hat{\Theta}_{w}
$$

Here, we assumed that $\operatorname{sgn} \hat{U}_{w}=\operatorname{sgn} \hat{\Theta}_{w}$. With (18), the empirical specification of $C_{f}(\mathrm{Ra}, \mathrm{Pr})$ is no longer necessary, and the model is given by

$$
\begin{gathered}
\frac{d \hat{U}_{w}}{d \hat{t}}=\frac{2 \hat{L}_{w}^{2}}{2 \hat{L}_{w}^{2}+1}\left(\frac{1-4 \hat{\lambda}_{\Theta}}{2 \hat{L}_{w}} \hat{\Theta}_{w}-4 \alpha\left|\hat{U}_{w}\right| \hat{U}_{w}\right), \\
\frac{d \hat{\Theta}_{w}}{d \hat{t}}=\frac{2 \hat{\lambda}_{\Theta}}{\hat{L}_{w}} \hat{U}_{w}-\frac{4 \alpha}{\hat{L}_{w}^{2} \operatorname{Pr}_{T}}\left|\hat{U}_{w}\right| \hat{\Theta}_{w}-\frac{2}{\hat{\lambda}_{\Theta} \operatorname{Re}_{f} \operatorname{Pr}} \hat{\Theta}_{w} .
\end{gathered}
$$

The steady state solution of the model as a function of $\mathrm{Ra}$ is shown in Fig. 8. At this point, the only empirical data used in the model is the roll size $L_{w}$ and the power law for $\lambda_{\Theta}$ and the roll size $L_{w}$. The mixing parameter $\alpha$ is kept at the same value as in [9], namely, 0.6. As can be seen, the model captures the trends of $\hat{U}_{w}, \hat{\Theta}_{w}$, and $C_{f}$ satisfactorily. Note that the profiles could be made to match quantitatively as well if some additional coefficients were introduced. However, the focus of this paper is not to develop a carefully tuned model, but to elicit general scaling behavior.

It is not very useful to have an expression for $C_{f}$ in terms of $\hat{\Theta}_{w}$, as this quantity is rarely measured. However, by using the steady state solution of (19), $\hat{\Theta}$ can be expressed in terms of $\hat{U}_{w}$ as 


$$
\hat{\Theta}_{w}=\frac{8 \alpha \hat{L}_{w}}{1-4 \hat{\lambda}_{\Theta}}\left|\hat{U}_{w}\right| \hat{U}_{w},
$$

so that the $\hat{\Theta}_{w}$ dependence of $C_{f}$ can be eliminated. Using (18) and (21), $C_{f}$ is given by

$$
C_{f} \approx \frac{16 \alpha}{1-4 \hat{\lambda}_{\Theta}} \hat{\lambda}_{\Theta}
$$

Hence, when $\hat{\lambda}_{\Theta} \ll 1$, the model predicts that $C_{f} \propto \hat{\lambda}_{\Theta}$. Note that the $\lambda_{\Theta}$ term in the denominator represents the effects of wall friction. Hence, when $\hat{\lambda}_{\Theta} \ll 1, C_{f}$ scales independently of wall effects. It is the turbulence in the outer flow which fully determines the velocity at the edge of the boundary layer.

A scaling relation for $\lambda_{u}$ can be derived by using the two different expressions for $C_{f},(8)$ and (17). This results in

$$
2 \operatorname{Re}^{-1}\left(\frac{\lambda_{u}}{H}\right)^{-1}=\frac{2 \hat{\lambda}_{\Theta}\left|\hat{\Theta}_{w}\right|}{\hat{L}_{w} \hat{U}_{w}^{2}} .
$$

Using $\operatorname{Re}=\left|\hat{U}_{w}\right| \operatorname{Re}_{f}, \hat{\lambda}_{u}$ is approximated by

$$
\hat{\lambda}_{u} \approx \frac{\hat{L}_{w}\left|\hat{U}_{w}\right|}{\hat{\lambda}_{\Theta}\left|\hat{\Theta}_{w}\right| \operatorname{Re}_{f}}
$$

Dropping the absolute signs and using $(21), \hat{\lambda}_{u}$ is given by

$$
\hat{\lambda_{u}} \approx \frac{1-4 \hat{\lambda}_{\Theta}}{8 \alpha \hat{\lambda}_{\Theta} \hat{U}_{w} \operatorname{Re}_{f}}=\frac{1-4 \hat{\lambda}_{\Theta}}{8 \alpha} \frac{1}{\hat{\lambda}_{\Theta} \operatorname{Re}} .
$$

Upon assuming that $\hat{\lambda}_{\Theta} \ll 1$, it follows that $\hat{\lambda}_{u}$ scales as $\hat{\lambda}_{u}$ $\propto \hat{\lambda}_{\Theta}^{-1} \mathrm{Re}^{-1}$. Figure 8(d) shows the prediction of the wind model for $\lambda_{u}$. Although the boundary layer thickness is underpredicted, the trend is in agreement with the DNS data. Given the simplicity (with only one calibration parameter $\alpha$ ), the model captures the trends of wind velocity, spatial temperature difference, friction factor, and kinematic boundary layer thickness satisfactorily.

\section{TURBULENT OR NOT?}

The apparently contradictory findings reported in the previous sections are quite intriguing. On the one hand, the results indicate that the kinematic boundary layer is turbulent. The deduced boundary layer equation (9) shows that forcing due to turbulent Reynolds stresses is significant, in particular outside the thermal boundary layer. Furthermore, the spectra in the bulk and in the boundary layers are nearly indistinguishable and show the existence of a continuous range of active scales in both space and time. Both are an indication of turbulence.

On the other hand, the results suggest that the kinematic boundary layer does not correspond to a classical turbulent boundary layer. The Reynolds numbers in the Ra range we consider $\left(\operatorname{Re} \approx 1500\right.$ at $\left.\mathrm{Ra}=10^{8}\right)$ are generally considered too low to sustain turbulence. Moreover, the friction factor $C_{f}$ for a classical forced boundary layer has a weak dependence on $\operatorname{Re}$ (reflecting the quadratic wall friction), and is dominated by the turbulent momentum flux from the free stream. For the boundary layers under consideration, the dominant contributor to $C_{f}$ is the pressure gradient (Sec. III F) and not the momentum flux. Consequently, $C_{f}$ has a significant Re dependence. The near-universal profiles (found in the present work especially for the two lower Ra numbers) as a function of Ra based on the outer variables $\lambda_{u}$ and $\widetilde{u}_{\max }$ (Fig. 2) are further evidence against a classical turbulent boundary layer: a turbulent boundary layer by definition cannot be universally scaled by outer variables.

The difference between classical forced turbulence boundary layers and a boundary layer of Rayleigh-Bénard convection may be best characterized by the way the turbulence is produced and redistributed. For forced flow cases, turbulence cannot be maintained at low Re, as the dissipation in the boundary layer will be stronger than the production. However, for Rayleigh-Bénard convection, the production and transport of turbulent kinetic energy (TKE) are not confined to the inner layer alone. Instead, TKE is produced in the bulk, where it is partially dissipated. The surplus is transported to the boundary layer by pressure velocity fluctuations (see also [22]). Therefore, there is no local equilibrium between production and dissipation, and turbulence can be maintained in the boundary layers below the critical Re. At sufficiently high $\mathrm{Ra}$, instabilities due to shear can be expected to maintain themselves, and several experiments and simulations show such a transition around $\mathrm{Ra}=10^{11}$ $[11,12,23]$.

A simple explanation for the laminarlike scaling of classical integral boundary layer parameters may be that the forcing in the wall-parallel direction is very weak compared to the forcing in the wall-normal direction (plume impingement and detachment). Indeed, the forcing in the vertical direction is the direct result of buoyancy, while the pressure gradient in the horizontal direction forms is due to largescale differences in mean temperature. This can be made explicit by considering the ratio of the forcing in the wallnormal direction (buoyancy) and wall-parallel direction (16), which is given by

$$
\frac{\beta g \Delta \Theta}{\beta g H L_{w}^{-1} \Theta_{w}}=\hat{L}_{w} \hat{\Theta}_{w}^{-1} .
$$

At $\mathrm{Ra}=10^{5}$, this ratio is approximately 50 , and at $\mathrm{Ra}=10^{7}$, the ratio is approximately 100 . Thus, the boundary layers under consideration here are forced primarily in the wallnormal direction, and the force generating the wind is relatively weak.

Despite the laminarlike scaling of the integral parameters, a parallel can be drawn with a fully developed forced boundary layer: both have a viscous sublayer dominated by viscosity which suppresses instabilities and prevents their growth and development of turbulence. However, as demonstrated by seminal experiments in the 1960s [24], despite linear velocity variation, the flow within the sublayer in a forced boundary layer is not laminar, but accompanied by considerable irregular fluctuations, streaks, and other structures. One 
can argue that the same dynamics occur in the boundary layers of Rayleigh-Bénard convection. In particular, Figs. 6(a)-6(c) indicate that the thermal boundary layer $0<z$ $<\lambda_{\Theta}$ functions as a viscous sublayer, and the region $\lambda_{\Theta}<z$ $<\lambda_{u}$ as a crossover region between the exterior flow and the thermal boundary layer. The absence of a constant-stress layer dominated by the turbulent momentum flux suppresses the logarithmic region and marks a fundamental difference from forced turbulent boundary layers.

\section{CONCLUSIONS}

The aim of this paper has been to study the boundary layers that develop under the joint action of plumes and wind in Rayleigh-Bénard convection at the top and bottom plates. Direct numerical simulation was used for simulations at Ra $=\left\{10^{5}, 10^{6}, 10^{7}, 10^{8}\right\}$ and $\operatorname{Pr}=1$ for $\Gamma=4$ aspect-ratio domains with periodic side boundary conditions. For each Ra, ten independent simulations were carried out, resulting in approximately 400 independent realizations per Ra. Processing the results using symmetry-accounting ensemble averaging made it possible to retain the wind structure, which would normally cancel out due to the translational invariance of the system.

The importance of Reynolds stresses in the boundary layers, as well as the temporal and spatial spectra, indicate undoubtedly a turbulent character of the boundary layer. However, the behavior is rather different from classical forced boundary layers, as can be judged from the laminarlike scaling of the classical integral boundary layer parameters. Indeed, viscous effects play an important role within the ther- mal boundary layer, and a large turbulent momentum flux from the external stream is absent. This difference is probably caused by the fact that the turbulence inside the kinematic boundary layer of Rayleigh-Bénard convection originates from the bulk, whereas classical forced boundary layers are in a local equilibrium between production and dissipation of turbulent kinetic energy.

Due to the importance of Reynolds stresses in the boundary layer, the arguments underpinning the kinematic boundary layer scaling $\lambda_{u} / H \propto \mathrm{Re}^{-1 / 2}$ do not hold. Using the DNS results and a conceptual wind model [9], explicit expressions for $C_{f}$ and $\lambda_{u}$ were derived. It was found that the friction factor should scale proportionally to the thermal boundary layer thickness as $C_{f} \propto \lambda_{\Theta} / H$. The kinematic boundary layer thickness scales inversely proportionally to the thermal boundary layer thickness and the Reynolds number as $\lambda_{u} / H \propto \operatorname{Re}^{-1}\left(\lambda_{\Theta} / H\right)^{-1}$. The predicted trends for $C_{f}$ and $\lambda_{u}$ are in agreement with the DNS results.

With the closure for $C_{f}$, the model (19) and (20) depends solely on empirical input for $\lambda_{\Theta}$, and predicts the wind Reynolds number Re, friction factor $C_{f}$, and kinematic boundary layer thickness $\lambda_{u}$.

\section{ACKNOWLEDGMENTS}

This work is part of the research program of the Stichting voor Fundamenteel Onderzoek der Materie (FOM), which is financially supported by the Nederlandse Organisatie voor Wetenschappelijk Onderzoek (NWO). The computations were sponsored by the Stichting Nationale Computerfaciliteiten $(\mathrm{NCF})$.
[1] S. Grossmann and D. Lohse, J. Fluid Mech. 407, 27 (2000).

[2] Y. B. Xin, K. Q. Xia, and P. Tong, Phys. Rev. Lett. 77, 1266 (1996).

[3] Y. B. Xin and K. Q. Xia, Phys. Rev. E 56, 3010 (1997).

[4] R. M. Kerr, J. Fluid Mech. 310, 139 (1996).

[5] R. M. Kerr and J. R. Herring, J. Fluid Mech. 419, 325 (2000).

[6] H. Schlichting and K. Gersten, Boundary Layer Theory (McGraw-Hill, New York, 2000).

[7] S. Grossmann and D. Lohse, Phys. Fluids 16, 4462 (2004).

[8] S. Grossmann and D. Lohse, J. Fluid Mech. 486, 105 (2003).

[9] M. van Reeuwijk, H. J. J. Jonker, and K. Hanjalić, preceding paper, Phys. Rev. E 77, 036311 (2007).

[10] X. Chavanne, F. Chilla, B. Castaing, B. Hebral, B. Chabaud, and J. Chaussy, Phys. Rev. Lett. 79, 3648 (1997).

[11] X. Chavanne, F. Chilla, B. Chabaud, B. Castaing, and B. Hebral, Phys. Fluids 13, 1300 (2001).

[12] G. Amati, K. Koal, F. Massaioli, K. R. Sreenivasan, and R. Verzicco, Phys. Fluids 17, 121701 (2005).

[13] J. Verdoold, M. van Reeuwijk, M. J. Tummers, H. J. J. Jonker, and K. Hanjalić, Phys. Rev. E 77, 016303 (2008).

[14] M. van Reeuwijk, H. J. J. Jonker, and K. Hanjalić, Phys. Fluids
17, 051704 (2005).

[15] F. M. White, Viscous Fluid Flow (McGraw-Hill, New York, 1991).

[16] S. Lam, X. D. Shang, S. Q. Zhou, and K. Q. Xia, Phys. Rev. E 65, 066306 (2002).

[17] X. L. Qiu and K. Q. Xia, Phys. Rev. E 58, 5816 (1998).

[18] R. B. Dean, J. Fluids Eng. 100, 215 (1978).

[19] N. Rajaratnam, Turbulent Jets, Developments in Water Science No. 5 (Elsevier, Amsterdam, 1976).

[20] J. J. Niemela and K. R. Sreenivasan, J. Fluid Mech. 557, 411 (2006).

[21] F. Heslot, B. Castaing, and A. Libchaber, Phys. Rev. A 36, 5870 (1987).

[22] R. M. Kerr, Phys. Rev. Lett. 87, 244502 (2001).

[23] J. J. Niemela and K. R. Sreenivasan, J. Fluid Mech. 481, 355 (2003).

[24] S. J. Kline, W. C. Reynolds, F. A. Schraub, and P. W. Runstadler, J. Fluid Mech. 30, 741 (1967).

[25] The values for $C_{f}$ in Table III are slightly smaller than those in Table I, as the latter are deduced from the plane-averaged squared mean velocity. 